\title{
Uniform alignment of non- $\pi$-conjugated species enhances deep ultraviolet optical nonlinearity
}

Jing Lu,${ }^{\dagger}$ Jia-Ning Yue, ${ }^{\dagger}$ Lin Xiong, ${ }^{\dagger}$ Wen-Kai Zhang, ${ }^{\star}$ Ling Chen,,${ }^{*} \dagger$ Li-Ming Wu* ${ }^{*} \dagger$

${ }^{\dagger}$ Beijing Key Laboratory of Energy Conversion and Storage Materials, College of Chemistry, Beijing Normal University, Beijing 100875, China

${ }^{\ddagger}$ Center for Advanced Quantum Studies, Department of Physics and Applied Optics Beijing Area Major Laboratory, Beijing Normal University, Beijing, 100875, China.

\section{EXPERIMENTAL SECTION}

Synthesis and Physical Properties of $\mathrm{NaNH}_{4} \mathrm{PO} 3 \mathrm{~F}_{3} \cdot \mathrm{H}_{2} \mathrm{O}$ (NNPF). High-purity NNPF was synthesized by dissolving $20.7 \mathrm{~g}$ mixture of $\mathrm{Na}_{2} \mathrm{PO}_{3} \mathrm{~F}$ and $\left(\mathrm{NH}_{4}\right)_{2} \mathrm{PO}_{3} \mathrm{~F}$ (synthesized according to literature ${ }^{1}$ ) in $25 \mathrm{ml}$ deionized water into a saturated solution. $250 \mathrm{ml}$ acetone was added to precipitate colourless polycrystalline powder of NNPF. The precipitate was filtrated, washed with acetone several times, and subsequently dried in an oven at $60^{\circ} \mathrm{C}$. The reaction is described by equation (1):

$$
\mathrm{Na}_{2} \mathrm{PO}_{3} \mathrm{~F}+\left(\mathrm{NH}_{4}\right)_{2} \mathrm{PO}_{3} \mathrm{~F}+2 \mathrm{H}_{2} \mathrm{O}=2 \mathrm{NaNH}_{4} \mathrm{PO}_{3} \mathrm{~F} \cdot \mathrm{H}_{2} \mathrm{O}
$$

The experimental powder X-ray diffraction (XRD) matched well with the simulated one and confirmed its phase purity (Fig. S1).

The density measurement of NNPF crystals was measured at $17{ }^{\circ} \mathrm{C}$ by the Buoyancy method. To evaluate the thermal stability of the NNPF crystal, TG-DSC curves were carried out on a NETZSCH STA 449F3 analyzer from $25-500{ }^{\circ} \mathrm{C}$ at a rate of $10 \mathrm{~K} / \mathrm{min}$ under the nitrogen atmosphere with an $\mathrm{Al}_{2} \mathrm{O}_{3}$ crucible containing the NNPF powder sample. The chemical stability of NNPF was studied at room temperature, a NNPF crystal with weight of $0.1235 \mathrm{~g}$ was placed in air for 5 months, and two thin crystals with weights of $0.1118 \mathrm{~g}$ and $0.0633 \mathrm{~g}$ were immerged into ethanol and acetone solution for 15 days.

Crystal Growth. Single crystals of NNPF were grown by a vapor-liquid diffusion method. To optimize the growth conditions, the solubility curve of NNPF crystal was 
studied from $30-60{ }^{\circ} \mathrm{C}$ by gravimetric method (Fig. S2). A seed crystal was obtained by slow evaporation of an aqueous solution at room temperature. Stoichiometric $\mathrm{Na}_{2} \mathrm{PO}_{3} \mathrm{~F}(6.420 \mathrm{~g}, 4.46 \mathrm{mmol})$ and $\left(\mathrm{NH}_{4}\right)_{2} \mathrm{PO}_{3} \mathrm{~F}(6 \mathrm{~g}, 4.47 \mathrm{mmol})$ were dissolved in 30 $\mathrm{mL} \mathrm{H}_{2} \mathrm{O}$ and stirred until clear and the filtrate was then collected, into which a NNPF seed crystal tightly attached on a platinum wire was suspended. Subsequently, the assembly was put inside a large beaker containing $150 \mathrm{ml}$ acetone to ensure the acetone diffusion. After 10 days, a transparent layer of acetone was seen on the top of the saturated solution, and then the NNPF solution was gradually grew as cloudy as milk. After 24 days, an NNPF crystal with dimensions of $14 \times 12 \times 2.8 \mathrm{~mm}^{3}$ was pulled out from the solution.

Characterization. Powder X-ray Diffraction (PXRD) was collected at room temperature on Bruker-AXS D8 Advance X-ray diffractometer, using $\mathrm{Cu} \mathrm{K} \alpha_{1}$ radiation

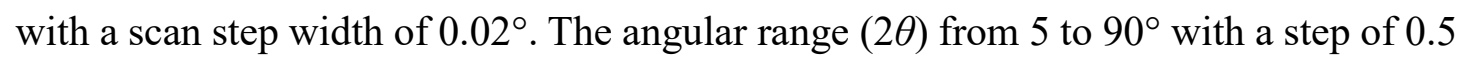
s. An X-ray orientator (Dandong Co., model: YX-2) and the corresponding XRD patterns were used to examine the major facets of the NNPF single crystal. Raman spectrum was recorded in the range of $100-5000 \mathrm{~cm}^{-1}$ using a LabRAM HR Evolution Raman spectrometer of Horiba employing the $532 \mathrm{~nm}$ excitation laser with laser power of $100 \mathrm{~W}$ at $298 \mathrm{~K}$. And a $50 \times$ objective lens was used to focus on the samples with a gratings of 100 lines/mm and acquisition time was $5 \mathrm{~s}$. The graphing software OriginPro (OriginLab) was used to obtain the full width at half maximum ${ }^{2}\left(\mathrm{FWHM}^{5}\right)$ through Gaussian fitting curve and excellent goodness of fit were reached in all samples $(R-$ squared above 0.978).

${ }^{31} \mathrm{P}$ and ${ }^{19} \mathrm{~F}$ NMR Spectra were recorded on a JNM-ECZ600R $600 \mathrm{M}$ spectrometer at with a rotation of $12 \mathrm{kHz}$. Polycrystalline powder were dissolved in $\mathrm{D}_{2} \mathrm{O}$ and in a FEP NMR tubes for NMR measurements.

Second Harmonic Generation (SHG) Measurement On Single Crystals with Relatively Small Size.

The $1030 \mathrm{~nm}$ pulses (290 fs, $240 \mathrm{kHz}$ ) emitted from Yb:KGW femtosecond laser (Pharos) were used as fundamental light. Optical parametric amplifier (ORPHEUS-HP, Light Conversion) was used to generate tunable excitation pulses. The SHG generated 
passed through a Fiber-based spectrometers (Thorlabs' fiber-based, compact, zernyTurner CCD spectrometers, a wide 200-1000 nm spectral range with better than $2 \mathrm{~nm}$ accuracy $)$ and recorded by a computer. A simple-polished single crystal $(12 \times 10 \times 1.8$ $\mathrm{mm}^{3}$ ) was put on an antioxidant aluminum sample holder and manually rotated during the test to let the fundamental light hit the crystal at different angles, made it exactly reach the phase-matching condition. The SHG output could be seen on the photographic card, the best matching state was achieved when the multiplier light was shrunk into a bright light spot from a scattered one. The energy meter was used to record the power of the SHG output and the fundamental light.

Single Crystal Laser-Induced Damage Threshold Measurement (LIDT). The single crystal LIDT measurement was carried out using a Nd:YAG nanosecond laser (Minilite II, Continuum Eletro-Optics, Inc.) at $1064 \mathrm{~nm}$ with a pulse duration of $10 \mathrm{~ns}$. The laser beam was focused on the crystal by a lens. An as-grew (010) crystal plate with a size of $6 \times 3.8 \times 0.60 \mathrm{~mm}^{3}$ was hand-polished with sand paper and loaded into the sample holder, and a KDP crystal plate (The KDP was bought from Core Optronics Co.,Ltd, Flatness lambda:/8@633nm; Surface finish: 10/5 Srcatch/Dig) with dimension of $8 \times$ $8 \times 0.43 \mathrm{~mm}^{3}$ was used as the reference. The spot diameter were 1.89 and $1.74 \mathrm{~mm}$ while the laser energy were $150 \mathrm{~mJ}$ for NNPF and KDP, respectively. LIDT is defined as LIDT $=E /(S \times P W), E, S, P W$ are laser energy, the beam area and pulse duration, respectively. The damage was confirmed afterwards under a microscope by observing the irradiated sites.

Refractive Index Measurements. Immersion technique ${ }^{3-4}$ was performed to determine the birefringence with the aid of an FGR-0002 Gem Refractometer on a polished (010)-NNPF and (011)- $\left(\mathrm{NH}_{4}\right)_{2} \mathrm{PO}_{3} \mathrm{~F}$ crystal plates with a thickness of $1.1 \mathrm{~mm}$. Using sodium yellow light $(589.3 \mathrm{~nm})$ as light source. The maximum and minimum values were measured at different angles by rotating crystal in the plane (Table S4). 


\section{Tables and Figures.}

Table S1. Crystallographic data and structure refinement of NNPF.

\begin{tabular}{|c|c|}
\hline Compound & $\mathrm{NaNH}_{4} \mathrm{PO}_{3} \mathrm{~F} \cdot \mathrm{H}_{2} \mathrm{O}$ \\
\hline Formula weight & 157.02 \\
\hline Crystal system & Monoclinic \\
\hline Crystal color & colorless \\
\hline Space group & $P c$ (No. 7) \\
\hline$a(\AA)$ & $6.0515(6)$ \\
\hline$b(\AA)$ & $9.0167(8)$ \\
\hline $\mathrm{c}(\AA)$ & $4.9402(7)$ \\
\hline$\beta$ (deg.) & $90.737(1)$ \\
\hline$V\left(\AA^{3}\right)$ & 269.54 \\
\hline Z & 2 \\
\hline$D_{c}\left(g \cdot \mathrm{cm}^{-3}\right)$ & 1.93 \\
\hline$\mu\left(\mathrm{mm}^{-1}\right)$ & 0.5 \\
\hline$F(000)$ & 160.0 \\
\hline GOOF on $F^{2}$ & 1.294 \\
\hline Flack parameter & $0.02(4)$ \\
\hline$R_{1}, w R_{2}(I>2 \sigma(I))^{a}$ & $0.0222,0.0571$ \\
\hline$R_{1}, w R_{2}(\text { all data })^{a}$ & $0.0229,0.0574$ \\
\hline
\end{tabular}

Table S2. Selected hydrogen bonds $(\AA)$ of $\mathrm{N}-\mathrm{H}{ }^{\cdots} \mathrm{O}$ and $\mathrm{O}_{\mathrm{w}}-\mathrm{H}^{\cdots} \mathrm{O}$ in $\mathrm{NaNH}_{4} \mathrm{PO}_{3} \mathrm{~F} \cdot \mathrm{H}_{2} \mathrm{O}(\mathrm{NNPF})$ compound.

\begin{tabular}{ccccc}
\hline $\mathbf{D}-\mathbf{H} \cdots \mathbf{A}$ & $\mathbf{D}-\mathbf{H}$ & $\mathbf{H} \cdots \mathbf{A}$ & $\mathbf{D} \cdots \mathbf{A}$ & $\mathbf{D}-\mathbf{H} \cdots \mathbf{A}$ \\
\hline $\mathrm{N} 1-\mathrm{H} 1 \cdots \mathrm{O} 3^{\mathrm{i}}$ & $0.971(5)$ & $1.914(9)$ & $2.875(5)$ & $169.4(9)$ \\
$\mathrm{N} 1-\mathrm{H} 2 \cdots \mathrm{O} 3{ }^{\mathrm{ii}}$ & $0.971(3)$ & $1.907(4)$ & $2.857(7)$ & $165.3(7)$ \\
$\mathrm{N} 1-\mathrm{H} 3 \cdots \mathrm{O} 3^{\mathrm{iii}}$ & $0.970(7)$ & $1.942(7)$ & $2.903(2)$ & $169.8(2)$ \\
$\mathrm{N} 1-\mathrm{H} 4 \cdots \mathrm{O} 2^{\mathrm{iv}}$ & $0.969(4)$ & $2.155(0)$ & $3.084(6)$ & $160.1(8)$ \\
$\mathrm{O}_{\mathrm{w}} 4-\mathrm{H} 5 \cdots \mathrm{O} 1^{\mathrm{i}}$ & $0.797(5)$ & $2.065(0)$ & $2.831(3)$ & $161.0(2)$ \\
$\mathrm{O}_{\mathrm{w}} 4-\mathrm{H} 6 \cdots \mathrm{O} 2^{\mathrm{iv}}$ & $0.800(1)$ & $2.078(8)$ & $2.833(7)$ & $157.2(7)$ \\
\hline
\end{tabular}

Symmetry codes: (i) $\mathrm{x}+1 / 2,-\mathrm{y}+1, \mathrm{z}+1 / 2$; (ii) $\mathrm{x}, \mathrm{y}, \mathrm{z}$; (iii) $\mathrm{x}, \mathrm{y}, 1+\mathrm{z}$; (iv) $\mathrm{x}-1 / 2,-\mathrm{y}+1, \mathrm{z}+1 / 2$ 
Table S3. Bader atomic charges of selected monofluorophosphates and phosphates.

\begin{tabular}{|c|c|c|c|c|c|c|c|}
\hline \multirow{2}{*}{ System } & \multicolumn{4}{|c|}{ Cation } & \multicolumn{3}{|c|}{$\begin{array}{c}\text { Anion group } \\
\left(\mathrm{PO}_{3} \mathrm{~F} / \mathrm{PO}_{4}\right)\end{array}$} \\
\hline & $\mathrm{NH}_{4}$ & $\mathrm{Na} / \mathrm{Li} / \mathrm{Rb} / \mathrm{Cs}$ & K & $\mathrm{H}$ & $\mathrm{P}$ & $\mathrm{O}$ & $\mathrm{F}$ \\
\hline $\mathrm{NaNH}_{4} \mathrm{PO}_{3} \mathrm{~F} \cdot \mathrm{H}_{2} \mathrm{O}$ & 0.80 & 0.89 & & & 3.68 & -1.50 & -0.83 \\
\hline$\left(\mathrm{NH}_{4}\right)_{2} \mathrm{PO}_{3} \mathrm{~F}$ & 0.80 & & & & 3.67 & -1.48 & -0.83 \\
\hline $\mathrm{LiRb}_{2} \mathrm{PO}_{4}$ & & Li: 0.88; Rb: & & & 3.63 & -1.56 & \\
\hline $\mathrm{LiCs}_{2} \mathrm{PO}_{4}$ & & Li: 0.89; Cs: & & & 3.62 & -1.55 & \\
\hline
\end{tabular}

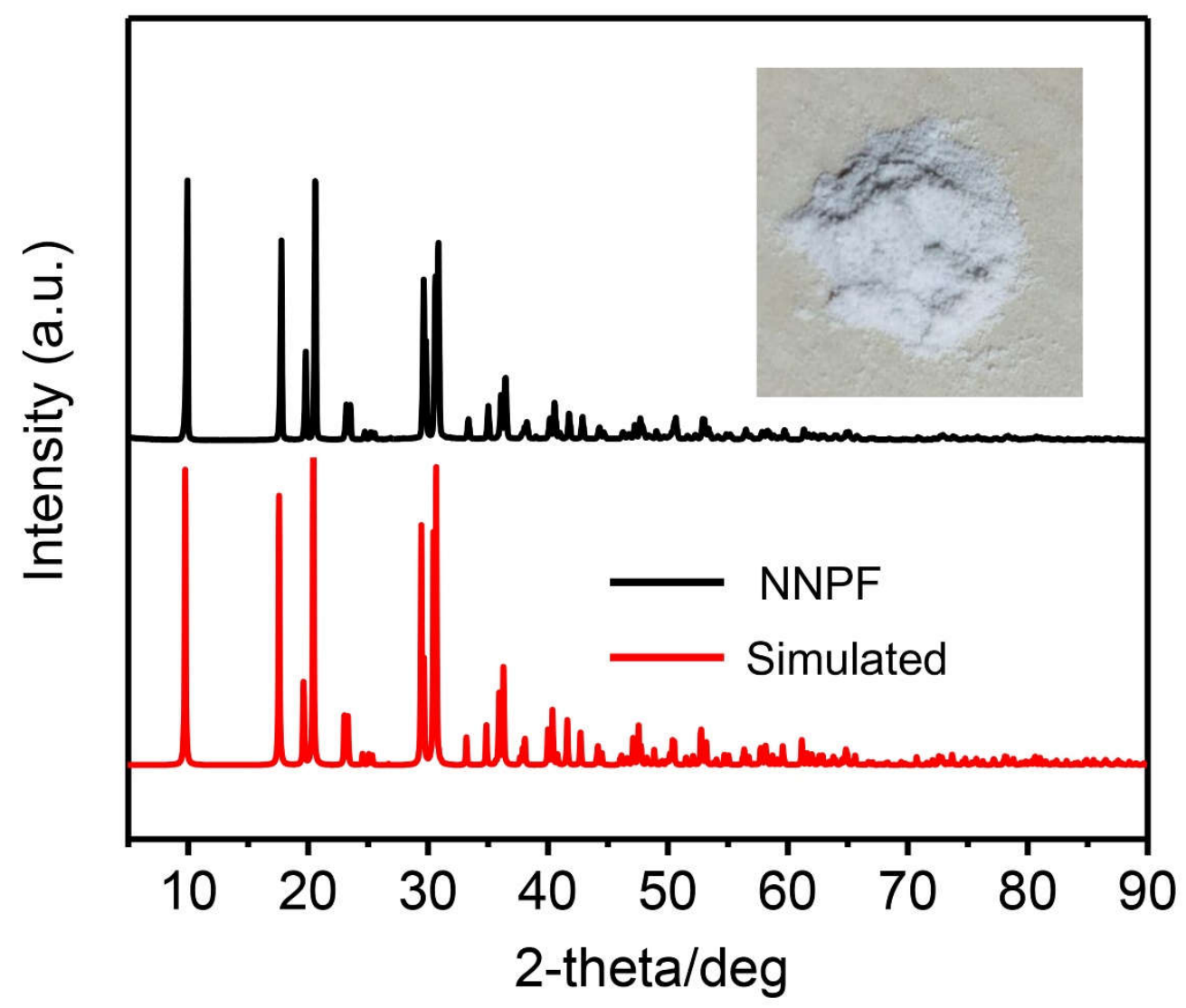

Figure S1. PXRD patterns of NNPF and the theoretical one indicating purity of the sample. Inset, the photo of the as-synthesized NNPF powder. 


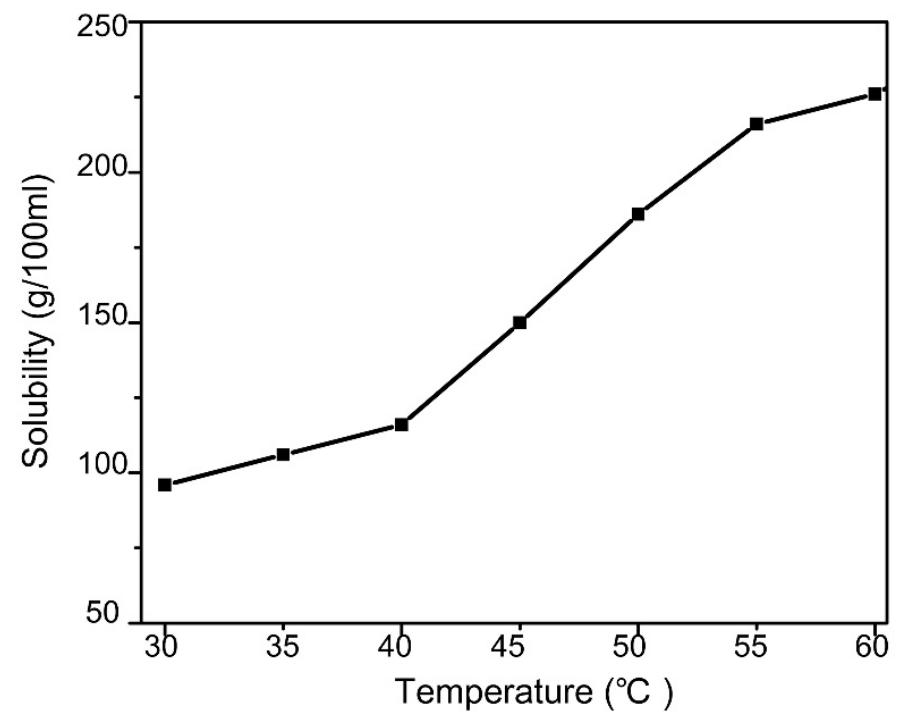

Figure S2. The solubility curve of NNPF polycrystalline powder from $30-60{ }^{\circ} \mathrm{C}$ in water.
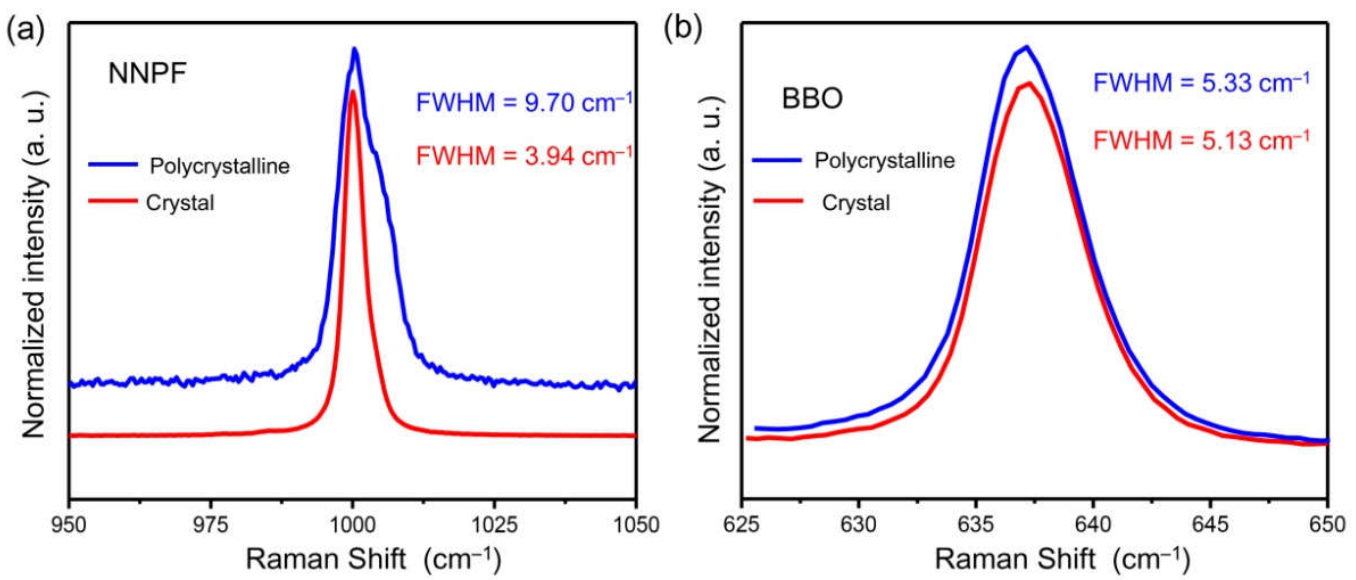

Figure S3. The FWHM of Raman spectra taken on single crystal and polycrystalline. a) NNPF, b) $\mathrm{BBO}$, used as references. 

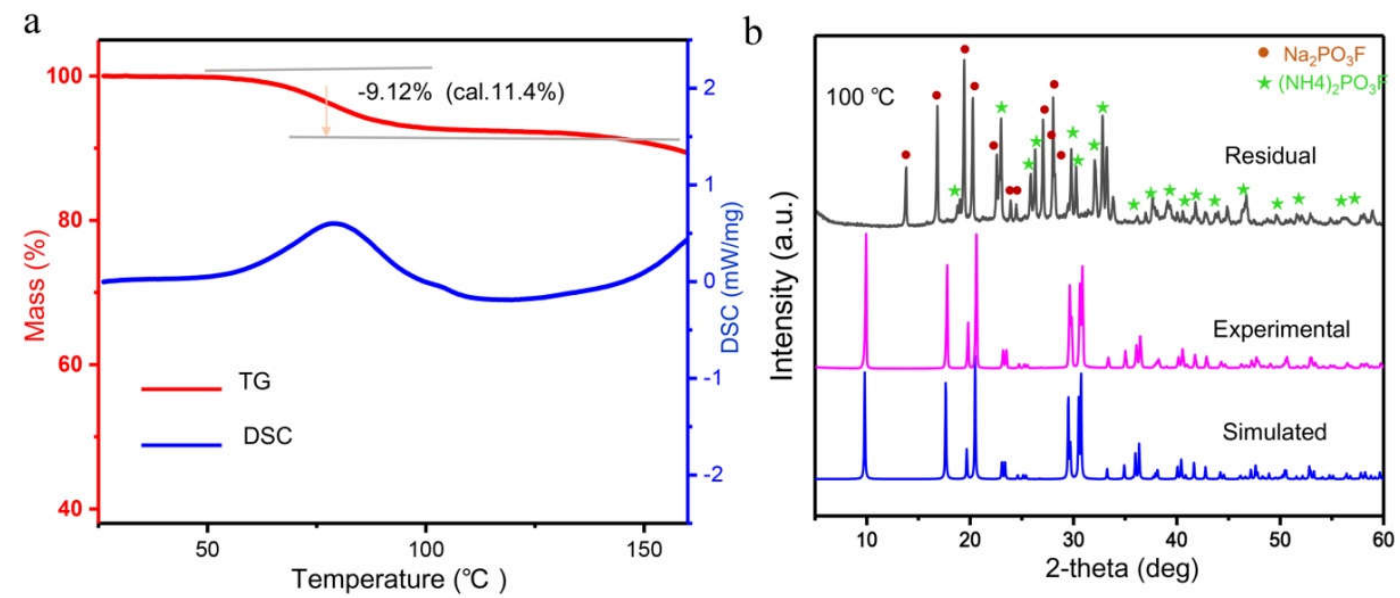

Figure S4. DSC, TG curves and the PXRD patterns for NNPF before and after the thermal measurements, decomposition of NNPF is seen.

a

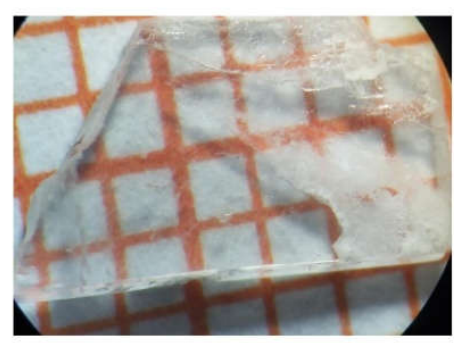

$\mathrm{C}$

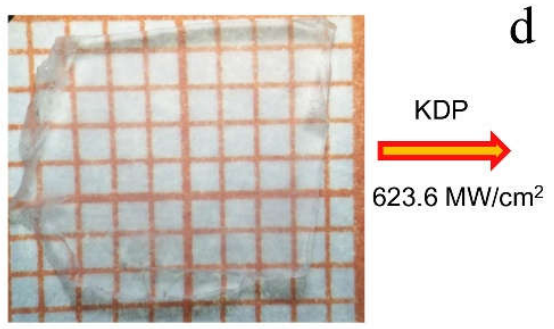

b

\section{NNPF}

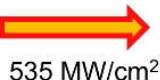

$535 \mathrm{MW} / \mathrm{cm}^{2}$
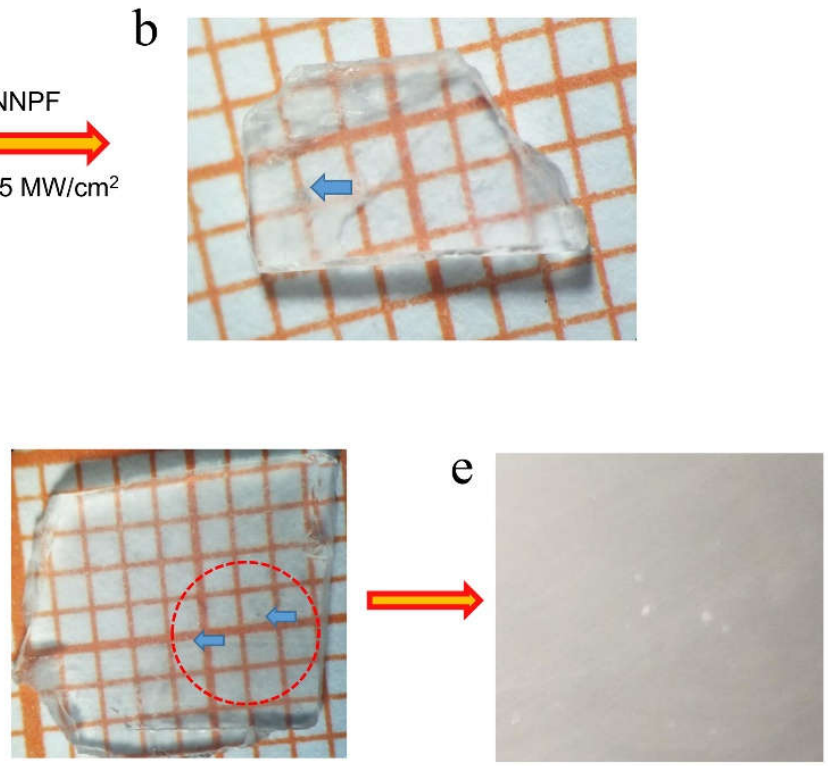

Figure S5. The measurement of LIDT on single crystals of a) NNPF $\left(6 \times 3.8 \times 0.60 \mathrm{~mm}^{3}\right)$ and c) KDP $\left(8 \times 8 \times 0.43 \mathrm{~mm}^{3}\right)$. After the measurement, the NNPF crystal surface b) was blackened and d, e) the KDP surface was indented (observed under the microscope). 
a

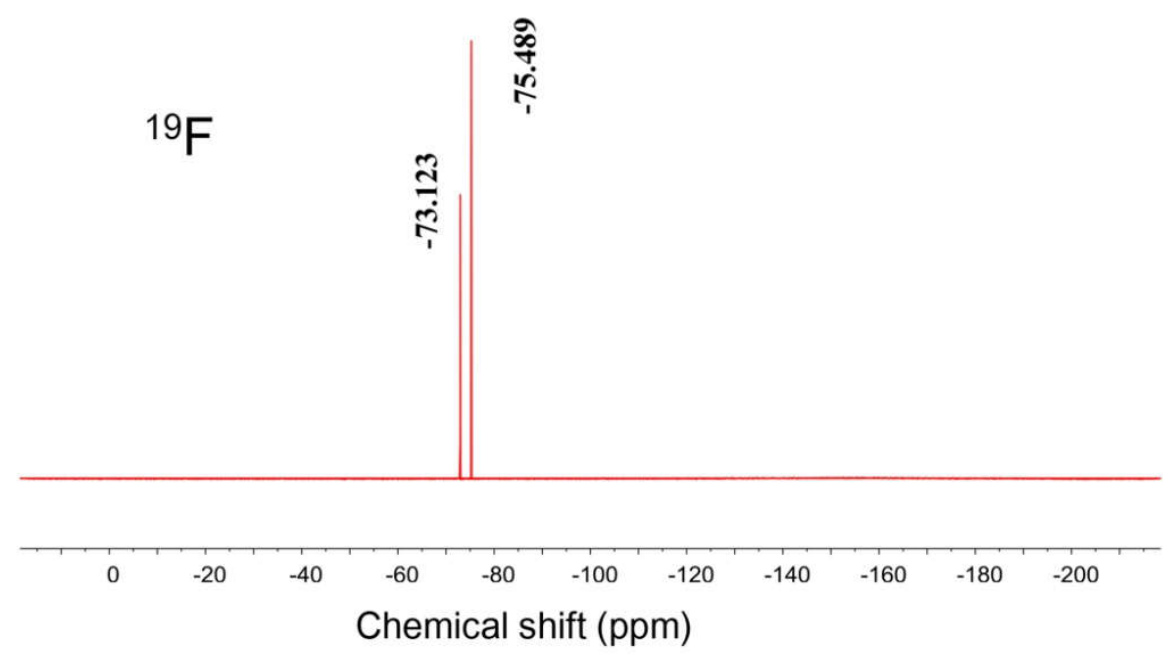

b

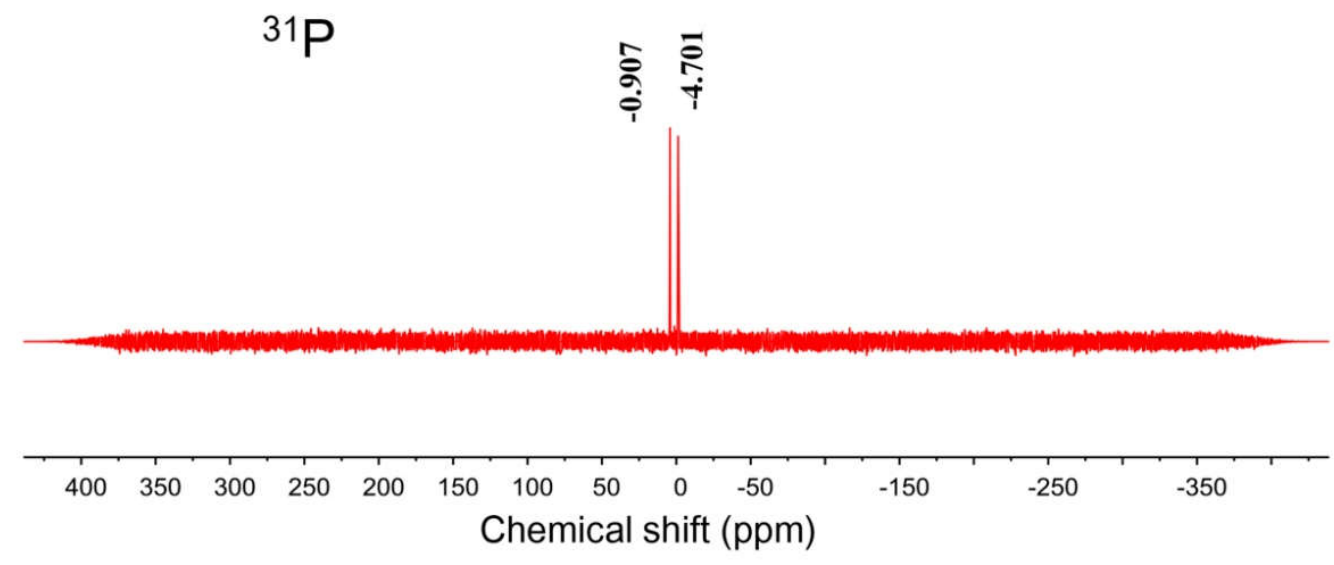

Figure S6. a) ${ }^{19} \mathrm{~F}$ and b) ${ }^{31} \mathrm{P}$ NMR of NNPF. 


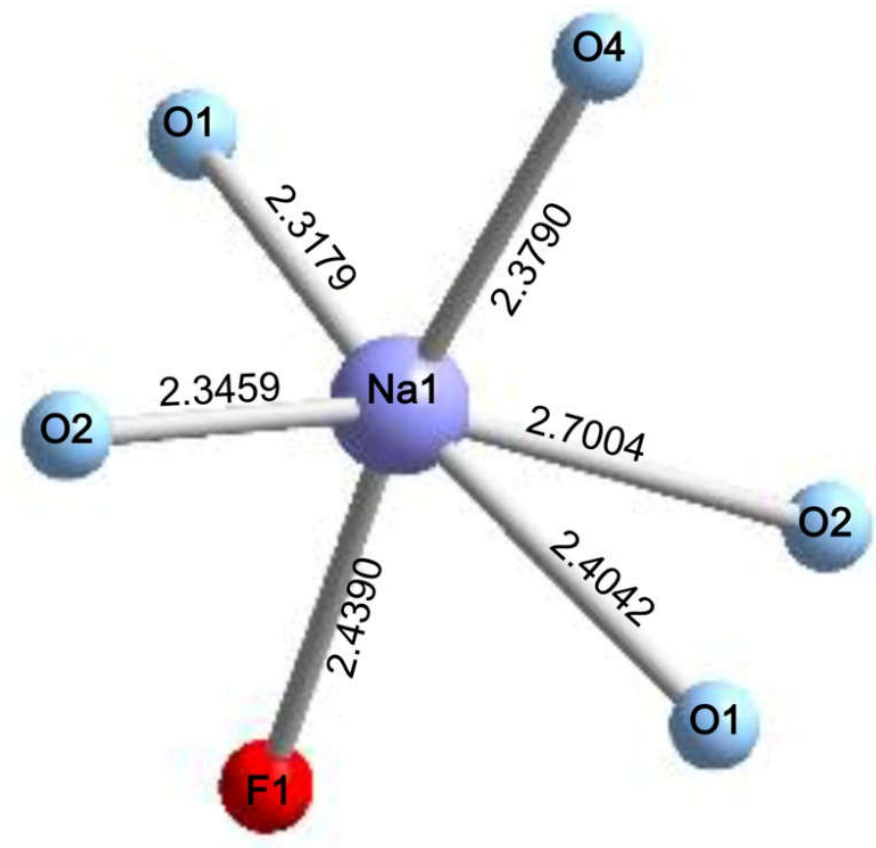

Figure S7. The distorted octahedron coordination environment of $\mathrm{Na}$ in NNPF. The Na atom is surrounded by $\mathrm{O} 4$ from $\mathrm{H}_{2} \mathrm{O}, \mathrm{O} 1-2$ and $\mathrm{F} 1$ atoms from three different $\left[\mathrm{PO}_{3} \mathrm{~F}\right]$ group.

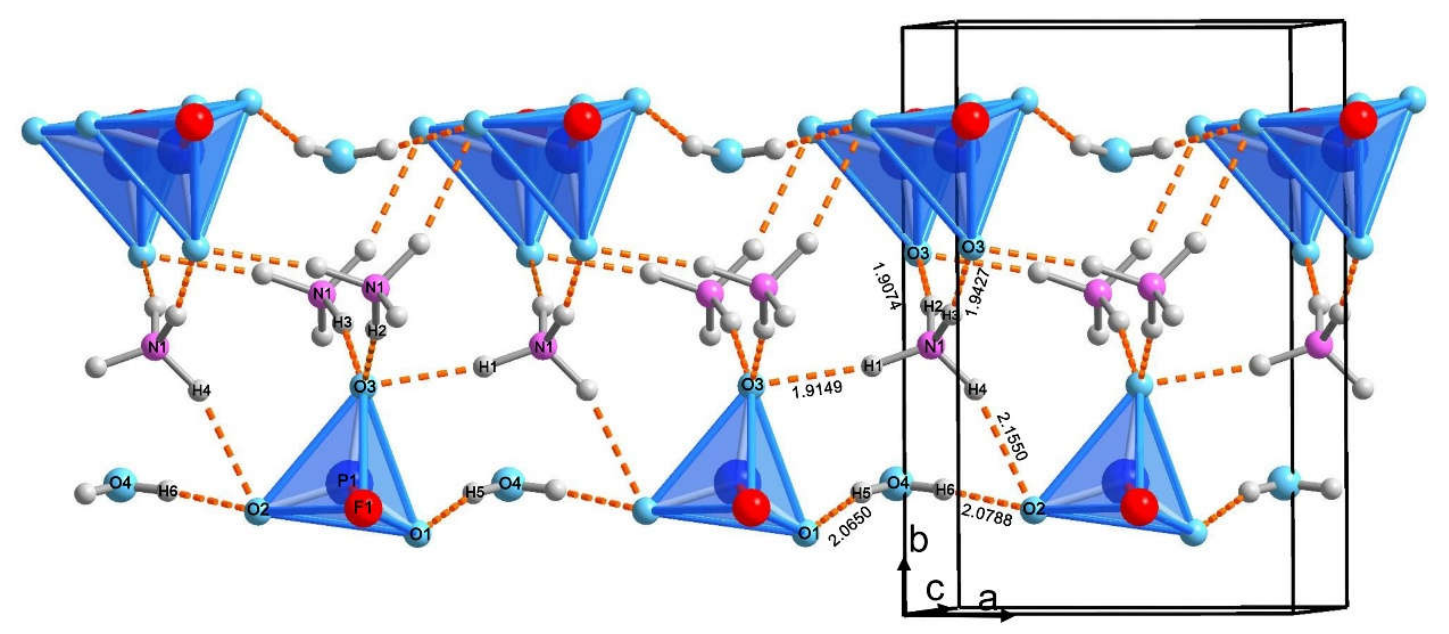

Figure S8. The hydrogen bonds framework in NNPF. O4 is the water oxygen. The yellow dashed line: $\mathrm{O}_{\mathrm{w}}-\mathrm{H} \cdots \mathrm{O}$ and $\mathrm{N}-\mathrm{H} \cdots \mathrm{O}$ hydrogen bonds, respectively. red, gray, blue, pink and light blue: F, H, P, N and $\mathrm{O}$ atoms, respectively. Tetrahedron: $\mathrm{PO}_{3} \mathrm{~F}$. 


\section{Supporting Information}

Table S4. Experimental refractive index measured on Single Crystal plates at $589.3 \mathrm{~nm}\left(n_{\max }\right.$ and $n_{\min }$ are shown in red).

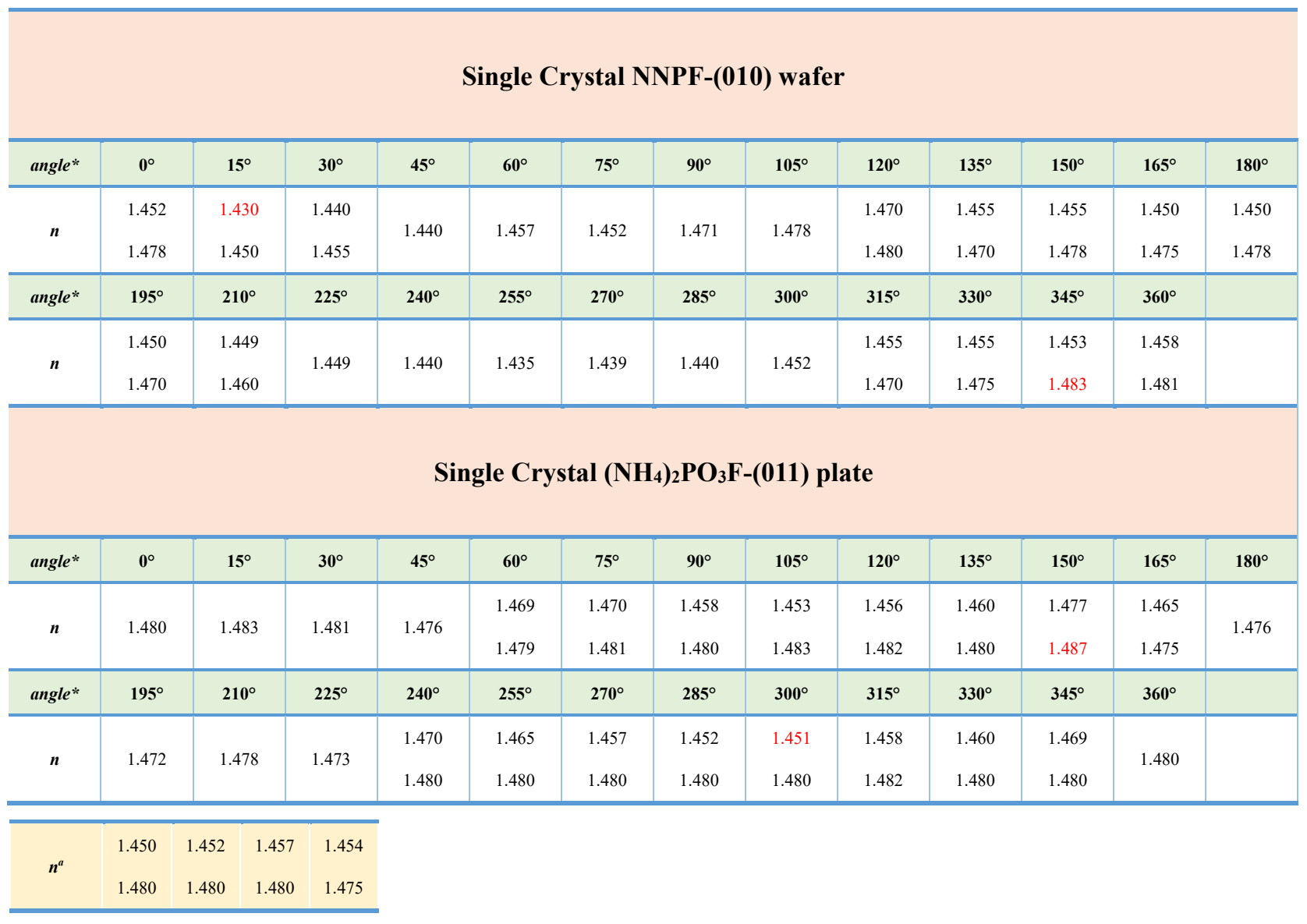

$n^{a}$ : Those reported for $\left(\mathrm{NH}_{4}\right)_{2} \mathrm{PO}_{3} \mathrm{~F}-(110)$ plate by ref 6 .

*angle: the definition is shown in Figure S9, left, inset. 

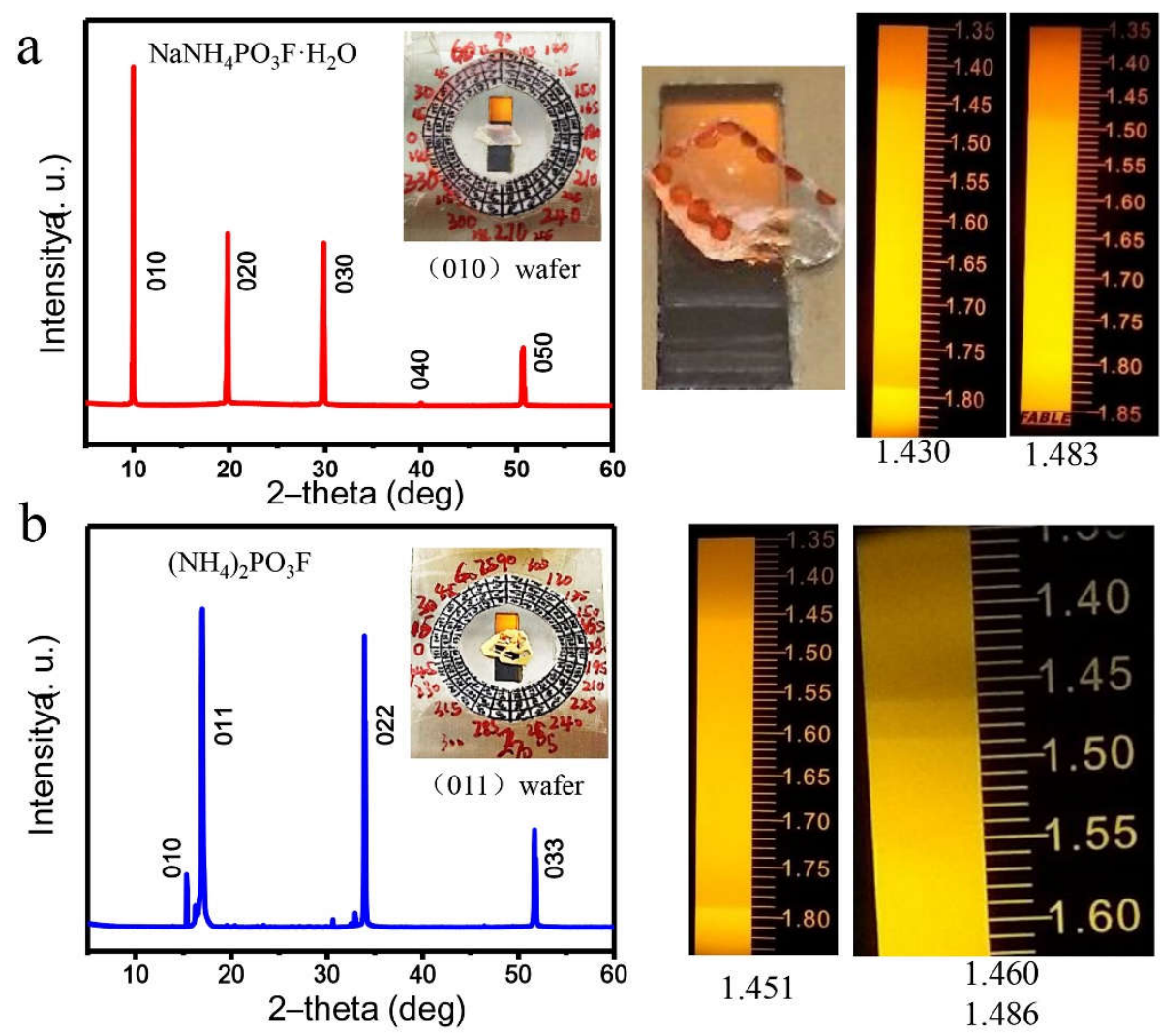

Figure S9. XRD patterns of NNPF and $\left(\mathrm{NH}_{4}\right)_{2} \mathrm{PO}_{3} \mathrm{~F}$ crystals and the photos showing the measured $n_{\max }$ and $n_{\min }$ of two crystals. The inset of the XRD patterns shows the definition of the rotation angle during the refractive index measurement (as listed in Table S4). The 1.79 in the photos (right) is the refractive index of the refraction oil. 


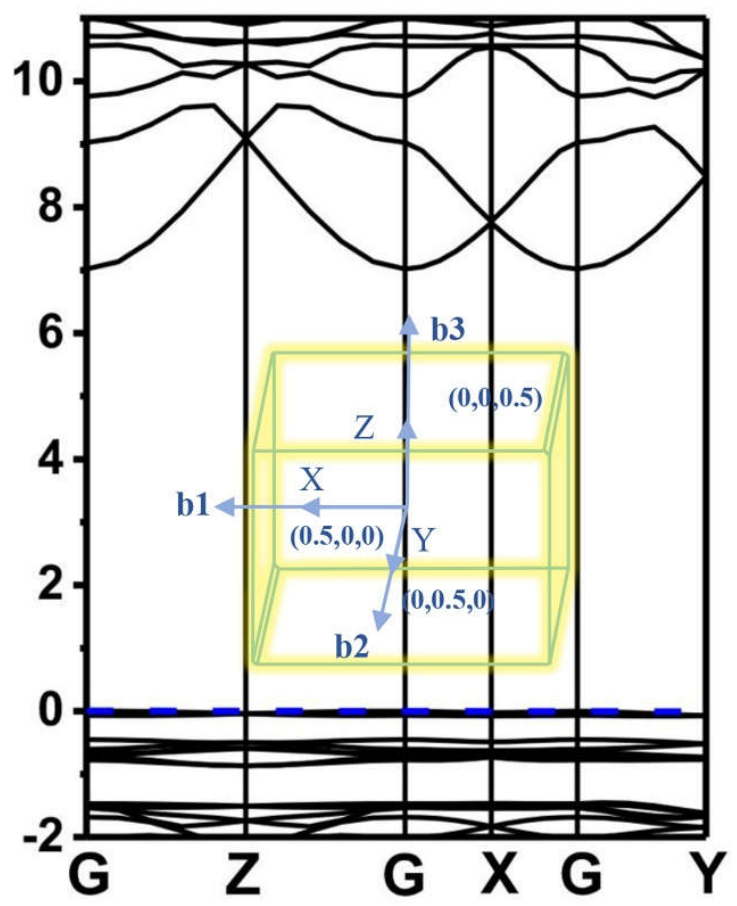

Figure S10. Band structure of NNPF. The dispersion difference along the $k$-paths of $\mathrm{G}(0,0,0)-\mathrm{Z}(0,0$, $0.5)$ and $\mathrm{G}-\mathrm{X}(0.5,0,0)$ is clear, inset: the Brillouin zone of NNPF lattice. 


\section{Supporting Information}

Table S5. Optical properties of typical phase-matching NLO crystals in UV and DUV region.

\begin{tabular}{|c|c|c|c|c|c|c|c|c|}
\hline Comp. & S.G. & $\begin{array}{c}\text { Cutoff } \\
\text { edge } \\
(\mathrm{nm})\end{array}$ & $\begin{array}{c}\text { SHG } \\
(\times \text { KDP })\end{array}$ & $d_{\mathrm{ij}}(\mathrm{pm} / \mathrm{V})$ & $\Delta n$ & $\begin{array}{l}\text { Shortest } \\
\lambda_{\mathrm{PM}}(\mathrm{nm})\end{array}$ & LIDT (GW) & Ref \\
\hline \multicolumn{9}{|c|}{ Borates } \\
\hline $\mathrm{LBO}\left(\mathrm{LiB}_{3} \mathrm{O}_{5}\right)$ & Pna $_{1}$ & 160 & 3 & $d_{31}=1.05$ & $0.0423 @ 589.3 \mathrm{~nm}$ & $\begin{array}{c}277 \\
\text { (obs.) }\end{array}$ & $\begin{array}{c}0.22 @ 532 \mathrm{~nm} \\
(10 \mathrm{~ns})\end{array}$ & 7,8 \\
\hline $\mathrm{CLBO}\left(\mathrm{CsLiB}_{6} \mathrm{O}_{10}\right)$ & $I \overline{4} 2 d$ & 180 & 2.2 & $d_{36}=0.95$ & $0.0521 @ 589.3$ nm & 213 & 15@1064nm (1 ns) & 9 \\
\hline $\mathrm{CBO}\left(\mathrm{CsB}_{3} \mathrm{O}_{5}\right)$ & $P 2_{1} 2_{1} 2_{1}$ & 167 & 2.7 & $d_{14}=1.08$ & $0.063 @ 1064$ nm & 273 & 26@1053 nm (1 ns) & 10 \\
\hline $\mathrm{BBO}\left(\beta-\mathrm{BaB}_{2} \mathrm{O}_{4}\right)$ & $R 3 c$ & 189 & 4 & $d_{22}=2.2$ & 0.118@589.3nm & $\begin{array}{c}213 \\
\text { (obs.) }\end{array}$ & $2.3 @ 1064$ nm $(10 \mathrm{~ns}, 10 \mathrm{~Hz})$ & 11 \\
\hline \multicolumn{9}{|c|}{ Fluoborates } \\
\hline $\begin{array}{c}\mathrm{KBBF} \\
\left(\mathrm{KBe}_{2} \mathrm{BO}_{3} \mathrm{~F}_{2}\right)\end{array}$ & $R 32$ & 147 & $\mathrm{~N} / \mathrm{A}$ & $\begin{array}{l}d_{11}=0.49 \\
d_{14}=0.76\end{array}$ & 0.072@1064 nm & $\begin{array}{c}173 \\
\text { (obs.) }\end{array}$ & $\begin{array}{c}8 @ 1064 \text { nm } \\
(0.035 \text { ns) }\end{array}$ & 12 \\
\hline $\mathrm{CsB}_{4} \mathrm{O}_{6} \mathrm{~F}$ & Pna $_{1}$ & 155 & 1.9 & $\begin{array}{c}\mathrm{d}_{32}=\mathrm{d}_{24}=0.92 \\
\mathrm{~d}_{33}=0.92\end{array}$ & 0.1137@1064 nm & 171.6 & $\mathrm{~N} / \mathrm{A}$ & 13 \\
\hline $\mathrm{NH}_{4} \mathrm{~B}_{4} \mathrm{O}_{6} \mathrm{~F}$ & Pna $_{1}$ & 156 & 3 & $\begin{array}{l}\mathrm{d}_{32}=1.07 \\
\mathrm{~d}_{33}=-1.19\end{array}$ & 0.1171@1064 nm & 164 & $\mathrm{~N} / \mathrm{A}$ & 14 \\
\hline \multicolumn{9}{|c|}{ Phosphates } \\
\hline $\mathrm{KLa}\left(\mathrm{PO}_{3}\right)_{4}$ & $P 2_{1}$ & 162 & 0.7 & $d_{\mathrm{ave}}=0.68$ & 0.0084@1064 nm & $>500$ & $\mathrm{~N} / \mathrm{A}$ & 15 \\
\hline $\mathrm{K}_{4} \mathrm{Mg}_{4}\left(\mathrm{P}_{2} \mathrm{O}_{7}\right)_{3}$ & $P c$ & 170 & 1.3 & N/A & $\begin{array}{c}0.0108 @ 1064 \text { nm } \\
\text { (obs.) }\end{array}$ & 494 & $\begin{array}{c}7 @ 1064 \text { nm } \\
(6 n s)\end{array}$ & 16 \\
\hline $\mathrm{Ba}_{2} \mathrm{NaClP}_{2} \mathrm{O}_{7}$ & $P 4 b m$ & $<176$ & 0.9 & $d_{\mathrm{ave}}=1.11$ & 0.017@1064 nm & 456 & $\begin{array}{c}0.056 @ 532 \text { nm } \\
(10 \mathrm{~ns}, 10 \mathrm{~Hz})\end{array}$ & 17 \\
\hline $\mathrm{RTP}\left(\mathrm{RbTiOPO}_{4}\right)$ & Pna $_{1}$ & 350 & N/A & $d_{31}=3.3 \pm 0.6$ & 0.0884 & 532 & $0.2 @ 1064 \mathrm{~nm}(10 \mathrm{~ns}, 10 \mathrm{~Hz})$ & 20 \\
\hline
\end{tabular}




\section{Supporting Information}

\begin{tabular}{|c|c|c|c|c|c|c|c|c|}
\hline & & & & $\begin{array}{l}d_{32}=4.1 \pm 0.8 \\
d_{33}=17.1 \pm 4.3\end{array}$ & & & & \\
\hline $\mathrm{KTP}\left(\mathrm{KTiOPO}_{4}\right)$ & Pna $_{1}$ & 352 & $\mathrm{~N} / \mathrm{A}$ & $\begin{array}{l}d_{31}=1.4 \\
d_{32}=2.6 \\
d_{33}=10.7\end{array}$ & 0.0921@1064 nm & 526 & $1.4(8 \mathrm{~ns})$ & 21 \\
\hline $\mathrm{KDP}\left(\mathrm{KH}_{2} \mathrm{PO}_{4}\right)$ & $I \overline{4} 2 d$ & 176 & & $d_{\mathrm{ave}}=0.96$ & 0.034@1064 nm & 258 & $0.7-1.2$ & 18 \\
\hline $\operatorname{DKDP}\left(\mathrm{KD}_{2} \mathrm{PO}_{4}\right)$ & $I \overline{4} 2 d$ & $<200$ & N/A & $d_{36}=0.37$ & 0.0383(@670 nm) & 266 & 0.7 & 19 \\
\hline $\mathrm{ADP}\left(\mathrm{NH}_{4} \mathrm{H}_{2} \mathrm{PO}_{4}\right)$ & $I \overline{4} 2 d$ & 184 & 1.2 & $\begin{array}{l}d_{36}=0.47 \\
\text { Monoflu }\end{array}$ & $\begin{array}{l}0.0394 @ 1064 \mathrm{~nm} \\
\text { es }\end{array}$ & 262 & N/A & 20 \\
\hline$\left(\mathrm{NH}_{4}\right)_{2} \mathrm{PO}_{3} \mathrm{~F}$ & Pna $_{1}$ & $<177$ & 1 & $\begin{array}{l}d_{15}=0.14 \\
d_{24}=0.40 \\
d_{33}=-0.35\end{array}$ & $\begin{array}{c}0.027 @ 1064 \text { nm } \\
0.03 @ 589.3 \text { nm } \\
\text { (obs.) }\end{array}$ & 264 & $\mathrm{~N} / \mathrm{A}$ & 6,22 \\
\hline $\mathrm{NaNH}_{4} \mathrm{PO}_{3} \mathrm{~F} \cdot \mathrm{H}_{2} \mathrm{O}$ & $P c$ & $<176$ & 1.1 & $\begin{aligned} d_{11} & =-0.727 \\
d_{12} & =0.451 \\
d_{15} & =0.534 \\
d_{24} & =0.169 \\
d_{33} & =-0.333 \\
d_{13} & =0.060\end{aligned}$ & $\begin{array}{c}0.053 @ 589.3 \mathrm{~nm} \\
\text { (obs.) }\end{array}$ & 194 & $\begin{array}{c}0.535 @ 532 \text { nm } \\
(10 \mathrm{~ns}, 10 \mathrm{~Hz})\end{array}$ & This work \\
\hline
\end{tabular}

N/A $=$ not reported or not available, $\lambda$ PM: shortest phase-matching second harmonic wavelength 


\section{Supporting Information}

\section{References}

(1) Krupkova, R.; Fabry, J.; Císařová, I; Vaněk, P. Bis (ammonium) fluorophosphate at room temperature. Acta Crystallogr C. 2002, 58, i66-i68.

(2) Querido, W.; Ailavajhala, R.; Padalkar, M.; Pleshko, N. Validated approaches for quantification of bone mineral crystallinity using transmission fourier transform infrared (FT-IR), attenuated total reflection (ATR) FT-IR, and Raman spectroscopy. Appl. Spectrosc. 2018, 72, 1581-1593

(3) Saylor, C. P. A thin cell for use in determinging the refractive indices of crystal grains. J. Res. Natl. Bur. Stand. $1935,15,97$.

(4) Chen, C. T.; Wang, Y. B.; Xia, Y. N.; Wu, B. C.; Tang, D. G.; Wu, K. C.; Zhen, W. R.; Yu, L. H.; Mei, L. F. New development of nonlinear optical crystals for the ultraviolet region with molecular engineering approach. $J$. Appl. Phys. 1995, 77, 2268-2272.

(5) Kamimura, T.; Ono, R.; Yap, Y. K.; Yoshimura, M.; Mori, Y.; Sasaki, T. Influence of crystallinity on the bulk laser-induced damage threshold and absorption of laser light in $\mathrm{CsLiB}_{6} \mathrm{O}_{10}$ crystals. J. Appl. Phys. 2001, 40, L111. (6) Zhang, B. B.; Han, G. P.; Wang, Y.; Chen, X. L.; Yang, Z. H.; Pan, S. L. Expanding frontiers of ultraviolet nonlinear optical materials with fluorophosphates. Chem. Mater. 2018, 30, 5397-5403.

(7) Devi, K.; Parsa, S.; Ebrahim-Zadeh, M. Birefringent-multicrystal, single-pass, continuous-wave secondharmonic-generation in deep-ultraviolet. Nonlinear. Opt A. IV. SPIE, 2016, 9894, 98940R.

(8) Chen, C.; Wu, Y.; Jiang, A.; Wu, B.; You, G.; Li, R.; Lin, S. New nonlinear-optical crystal: $\mathrm{LiB}_{3} \mathrm{O}_{5} . J O S A B, \mathbf{1 9 8 9}, 6,616-621$.

(9) Mori, Y.; Kuroda, I.; Nakajima, S.; Sasaki, T.; Nakai, S. New nonlinear optical crystal: cesium lithium borate. Appl. Phys. Lett. 1995, 67, 1818-1820.

(10) Wu, Y. C.; Fu, P. Z.; Wang, J. X.; Xu, Z. Y.; Zhang, L.; Kong, Y. F.; Chen, C. T. Characterization of CsB33 $\mathrm{O}_{5}$ crystal for ultraviolet generation. Opt. Lett. 1997. 22, 1840-1842.

(11) Chang, L. B.; Wang, S. C.; Kung, A. H. Efficient compact watt-level deep-ultraviolet laser generated from a multi-kHz Q-switched diode-pumped solid-state laser system. Opt. Commun. 2002, 209, 397-401.

(12) Chen, C. T.; Wang, Y. B.; Xia, Y. N.; Wu, B. C.; Tang, D. G.; Wu, K. C.; Zhen, W. R.; Yu, L. H.; Mei, L. F. New development of nonlinear optical crystals for the ultraviolet region with molecular engineering approach. $J$. Appl. Phys. 1995, 77, 2268-2272.

(13) Wang, X. F.; Wang, Y.; Zhang, B. B.; Zhang, F. F.; Yang, Z.H.; Pan, S. L. CsB4O66: A congruent-melting deep-ultraviolet nonlinear optical material by combining superior functional units. Angew. Chem. Int. Ed. 2017. 


\section{Supporting Information}

$56,14119-14123$.

(14) Shi, G. Q.; Wang, Y.; Zhang, F. F.; Zhang, B. B.; Yang, Z. H.; Hou, X. L.; Pan, S. L. Poeppelmeier, K. R. Finding the next deep-ultraviolet nonlinear optical material: $\mathrm{NH}_{4} \mathrm{~B}_{4} \mathrm{O}_{6}$ F. J. Am. Chem. Soc. 2017, 139, $10645-$ 10648.

(15) Shan, P.; Sun, T.; Chen, H.; Liu, H.; Chen, S.; Liu, X.; Xu, J. Crystal growth and optical characteristics of beryllium-free polyphosphate, $\mathrm{KLa}\left(\mathrm{PO}_{3}\right)_{4}$, a possible deep-ultraviolet nonlinear optical crystal. Sci. Rep. 2016, 6, 25201.

(16) Yu, H. W.; Young, J. S.; Wu, H. P.; Zhang, W. G.; Rondinelli, J. M.; Halasyamani, P. S. $\mathrm{M}_{4} \mathrm{Mg}_{4}\left(\mathrm{P}_{2} \mathrm{O}_{7}\right)_{3}(\mathrm{M}=$ $\mathrm{K}, \mathrm{Rb}$ ): structural engineering of pyrophosphates for nonlinear optical applications. Chem. Mater. 2017, 29, 18451855

(17) Chen, J.; Xiong, L.; Chen, L.; Wu, L. M. $\mathrm{Ba}_{2} \mathrm{NaClP}_{2} \mathrm{O}_{7}$ : unprecedented phase matchability induced by symmetry breaking and its unique fresnoite-type structure. J. Am. Chem. Soc. 2018, 140, 14082-14086.

(18) Kliner, D. A.; Di Teodoro, F.; Koplow, J. P. Moore, S. W.; Smith, A. V. Efficient second, third, fourth, and fifth harmonic generation of a Yb-doped fiber amplifier. Opt. Commun. 2002, 210, 393-398.

(19) Barnes, N. P.; Gettemy, D. J.; Adhav, R. S. Variation of the refractive index with temperature and the tuning rate for KDP isomorphs. JOSA. 1982, 72, 895-898.

(20) Nikogosyan, D. N. In Nonlinear optical crystals: a complete survey. Springer. 2012, 139-267.

(21) Vanherzeele, H. Optimization of a cw mode-locked frequency-doubled Nd:LiYF4 laser. Appl. Opt. 1988, 27, $3608-3615$.

(22) Xiong, L.; Chen, J.; Lu, J.; Pan, C. Y.; Wu, L. M. Monofluorophosphate: a new source of deep-ultraviolent nonlinear optical materials. Chem. Mater. 2018. 30, 7823-7830 